\title{
Plastid Glycerol-3-phosphate Acyltransferase Enhanced Plant Growth and Prokaryotic Glycerolipid Synthesis in Brassica napus
}

\author{
Huiling Kang ${ }^{\dagger}$, Chenxi Jia ${ }^{\dagger}$, Nian Liu, Alfatih Alamin Alhussain Aboagla, Wenling Chen, \\ Wei Gong, Shaohua Tang and Yueyun Hong * \\ National Key Laboratory of Crop Genetic Improvement, Huazhong Agricultural University, \\ Wuhan 430070, China; 15927316487@163.com (H.K.); jiachenxi0701@163.com (C.J.); \\ galaxy_liu163@163.com (N.L.); alfatih.alamin2015@gmail.com (A.A.A.A.); \\ chenw1828@163.com (W.C.); 13163256438@163.com (W.G.); amy.feather@163.com (S.T.) \\ * Correspondence: hongyy@mail.hzau.edu.cn; Tel.: +86-027-8728-0545 \\ + These authors contribute the work equally.
}

Received: 22 June 2020; Accepted: 24 July 2020; Published: 27 July 2020

\begin{abstract}
Plastid-localized glycerol-3-phosphate acyltransferase (ATS1) catalyzes the first-step reaction in glycerolipid assembly through transferring an acyl moiety to glycerol-3-phosphate (G3P) to generate lysophosphatidic acid (LPA), an intermediate in lipid metabolism. The effect of ATS1 overexpression on glycerolipid metabolism and growth remained to be elucidated in plants, particularly oil crop plants. Here, we found that overexpression of BnATS1 from Brassica napus enhanced plant growth and prokaryotic glycerolipid biosynthesis. BnATS1 is localized in chloroplasts and an in vitro assay showed that BnATS1 had acylation activity toward glycerol 3-phosphate to produce LPA. Lipid profiling showed that overexpression of BnATS1 led to increases in multiple glycerolipids including phosphatidylglycerol (PG), monogalactosyldiacylglycerol (MGDG), phosphatidylcholine (PC), and phosphatidylinositol (PI), with increased polyunsaturated fatty acids. Moreover, increased MGDG was attributed to the elevation of 34:6- and 34:5-MGDG, which were derived from the prokaryotic pathway. These results suggest that BnATS1 promotes accumulation of polyunsaturated fatty acids in cellular membranes, thus enhances plant growth under low-temperature conditions in Brassica napus.
\end{abstract}

Keywords: glycerol-3-phosphate acyltransferase; lipid metabolism; prokaryotic glycerolipids; plant growth

\section{Introduction}

Glycerolipids including phospholipids and galactolipids are essential components of cellular membranes and signal molecules involved in diverse biological processes [1]. Glycerol-3-phosphate acyltransferase (GPAT) catalyzes the first-step reaction in glycerolipid assembly through transferring an acyl moiety to glycerol-3-phosphate (G3P), producing lysophosphatidic acid (LPA), which is further acylated at the sn-2 position of the G3P backbone by lysophosphatidic acid acyltransferase (LPAAT) to generate phosphatidic acid (PA) [2-4]. PA is an important intermediate in glycerolipid metabolism. PA is activated by cytidinediphosphate diacylglycerol synthase (CDS) to produce cytidinediphosphate diacylglycerol (CDP-DAG), which is a precursor for phosphatidylglycerol (PG) and phosphatidylinositol (PI) [5-7]. Alternatively, PA is dephosphorylated by PA phosphohydrolase (PAH) or lipid phosphate phosphatase (LPP) to produce diacylglycerol (DAG) [8,9], which subsequently reacts with cytidinediphosphate-choline (CDP-choline) and CDP-ethanolamine to produce phosphatidylcholine (PC) and phosphatidylethanolamine (PE), respectively $[1,10,11]$. DAG is also a substrate for 
galacotolipids such as monogalactosyldiacylglycerol (MGDG), digalactosyldiacylglycerol (DGDG) [12], and storage lipid triacylglycerol (TAG) as well [13].

In plants, fatty acids are synthesized de novo in chloroplasts, and are either retained in the chloroplasts for membrane lipid assembly via the prokaryotic pathway or exported to the endoplasmic reticulum (ER) for synthesis of membrane lipids and storage TAG via the eukaryotic pathway [1,2] (Supplementary Figure S1). Both PA and its dephosphorylated DAG can be produced via the two pathways $[2,14]$. The prokaryotic pathway-derived PA and DAG are retained in chloroplasts for the synthesis of PG and galactolipids [1]. The eukaryotic pathway-derived glycerolipids can reenter the chloroplasts, in the form of PA or presumably DAG produced from phospholipids, particularly PC, for the synthesis of thylakoid lipids [8,15-19]. The lipid molecular species derived from the two pathways are distinguishable. Glycerolipids originated from the prokaryotic pathway feature with a 16-carbon (C16) acyl moiety at the sn-2 position of the glycerol backbone, whereas those from the eukaryotic pathway have an 18-carbon (C18) acyl at the sn-2 position due to differing substrate selectivity of acyltransferases between the two pathways in plants [20,21] (Supplementary Figure S1). Thylakoid membrane lipids are derived from the two pathways depending on plant species or tissue.

The Arabidopsis genome contains 10 GPATs, which include a soluble GPAT, namely the plastidic acyl-acyl carrier protein (ACP) GPAT (ATS1) [3,4], and nine membrane-associated GPATs designated GPAT1 to GPAT9 localized to the ER or mitochondria [22-28]. Most GPAT family members, GPAT1 to GPAT8, catalyze the sn-2 acylation of G3P to produce LPA and are involved in the synthesis of extracellular lipids such as cutin and suberin [22-30]. Of these GPATs, GPAT9 is more closely related to animal GPAT3 and is responsible for the synthesis of TAG and phospholipids in the eukaryotic pathway in Arabidopsis [31,32]. ATS1 is localized in chloroplasts involved in the prokaryotic pathway. ATS1 utilizes acyl-acyl carrier protein (acyl-ACP) as a substrate for acylation at the sn-1 position of G3P to produce sn-1 acyl-LPA, which is subsequently acylated by plasidic LPAAT1 (also named as ATS2) to produce PA $[3,4,33]$. It was reported that ATS1 is essential for PG synthesis in plastids and loss of ATS1 led to a marked reduction in prokaryotic galactolipids in plants [3,4,34]. However, the effect of ATS1 overexpression on glycerolipid metabolism and growth remained to be elucidated in plants, particularly in crop plants. In this study, we found that overexpression of Brassica napus ATS1 (BnATS1) enhances plant growth and prokaryotic glycerolipid biosynthesis in Brassica napus.

\section{Results}

\subsection{BnATS1 Is Expressed in Various Tissues and BnATS1 Is Localized in Chloroplasts}

To investigate the temporal and spatial distribution of BnATS1 mRNA in Brassica napus, total RNA was extracted from various tissues and used for analysis by quantitative real-time PCR. BnATS1 (BnaA08g06960D) is expressed in various tissues including leaves, stems, roots, flowers, flower buds, siliques, developing seeds, developing silique walls, and mature seeds with expression being highest in young leaves and lowest in mature seeds (Figure 1A). The results suggest that BnATS1 is highly expressed in green tissues, particularly in actively growing leaf tissues. To further explore the subcellular localization and activity of BnATS1, the full-length BnATS1 coding sequence (CDS) was cloned from cultivar (cv.) Westar (Brassica napus L.) by reverse transcription PCR using mRNA from leaves as a template, and the resultant CDS was fused with GFP at the C-terminus and then transiently expressed in the epidermal cells of tobacco leaves by Agrobacterium infiltration. Green fluorescent BnATS1-GFP was overlaid with the red fluorescence of chloroplasts (Figure 2), suggesting that BnATS1 is localized to chloroplasts and may be involved in the prokaryotic pathway. To test whether BnATS1 encodes a GPAT, BnATS1 was expressed in E. coli cells in an enzymatic activity assay. The resulting protein was able to catalyze the acylation of G3P using 16:0-CoA as a substrate to produce LPA (Figure 1B). 
A

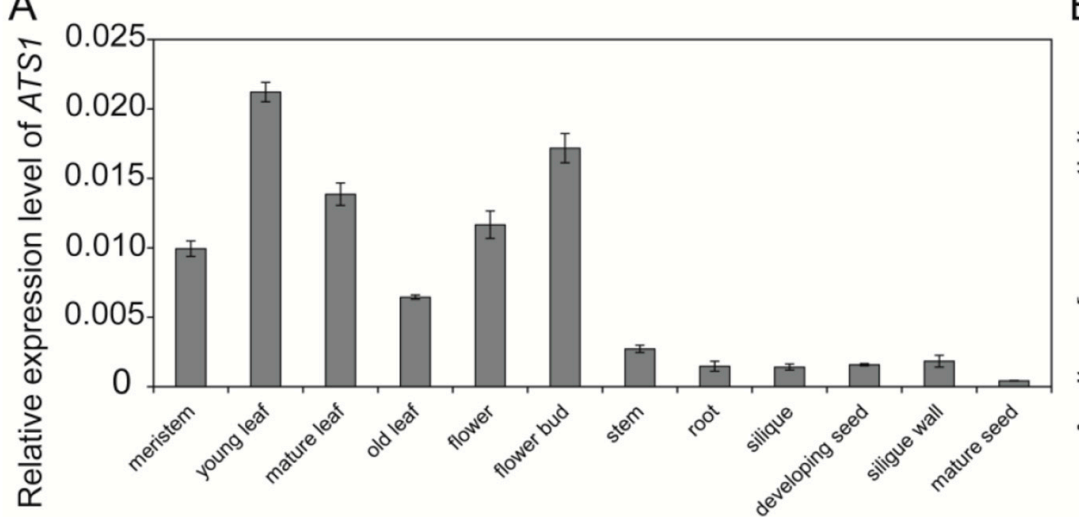

B

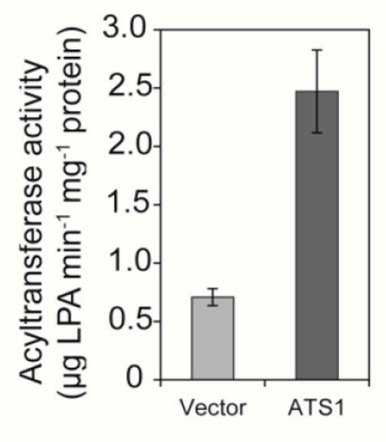

Figure 1. BnATS1 expression pattern and BnATS1 activity. (A) The expression pattern of BnATS1 in Brassica napus detected by RT-qPCR normalized to BnActin. Total RNA was extracted from various tissues of $\mathrm{cv}$. Westar. Values are means $\pm \mathrm{SD}$ ( $n=3$ separate samples). Leaves, roots, meristem, and stems were sampled from 40-day-old plants; flowers and flower buds were sampled from plants at the flowering stage; siliques, 10 days after pollination; developing seeds, 20 days after pollination; developing silique walls, 20 days after pollination; mature seeds, 45 days after pollination. (B) BnATS1 catalyzes the acylation of glycerol-3-phosphate using C16:0-CoA as an acyl donor to generate lysophosphatidic acid (LPA) in vitro. Values are means $\pm \mathrm{SD}(n=3$ independent experiments). LPA, lysophosphatidic acid.

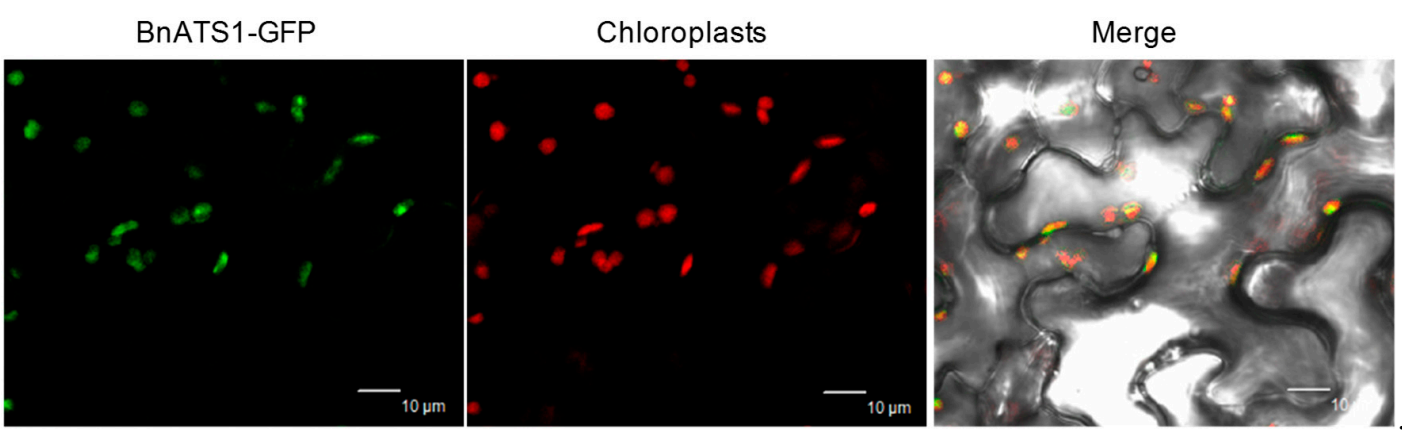

Figure 2. BnATS1 was localized in chloroplasts. BnATS1-GFP was transiently expressed in tobacco leaf cells under the control of the $35 S$ promoter. Green fluorescent signal of BnATS1-GFP was overlaid with red auto-fluorescence produced by chloroplasts observed using a confocal laser scanning microscope. Bars $=10 \mu \mathrm{m}$.

\subsection{Overexpression of BnATS1 Enhanced Plant Growth during Vegetative Stage}

BnATS1 is highly expressed in young leaves, suggesting a role in actively growing tissues. To investigate whether BnATS1 is involved in plant growth, BnATS1 was overexpressed in cv. Westar (Brassica napus L.) plants under the control of the CaMV $35 S$ promoter. More than 20 independent transgenic lines were obtained and BnATS1-overexpression (OE) plants exhibited a similar phenotype with enhanced vegetative growth in the field growth conditions, in which the temperature was approximately $7-10{ }^{\circ} \mathrm{C}$ (night)/ $15-20{ }^{\circ} \mathrm{C}$ (day) in fall and $0-3{ }^{\circ} \mathrm{C}$ (night)/7-12 ${ }^{\circ} \mathrm{C}$ (day) in winter (Figure $3 \mathrm{~A}$ ). We randomly selected three lines of them for functional characterization in detail. The BnATS1 transcript level in leaves of OE6, OE16, and OE22 plants was 9- to 33-fold higher than that of the wild type (WT) (Figure 3B). The plant height of BnATS1-OE was approximately 30\% greater relative to the WT at the vegetative stage (Figure 3C). The leaf length and width in BnATS1-OE plants were increased by approximately $34 \%$ and $29 \%$, respectively, as compared with the WT plants (Figure 3D,E). The fresh weight of the OE6, OE16, and OE22 lines was increased by 31\%, 50\%, and 38\%, respectively, as compared with the $\mathrm{WT}$ at the vegetative stage (Figure 3F). However, the leaf number of 
BnATS1-OE plants was comparable to that of WT plants (Figure 3G). Thus, enhanced fresh weight was primarily due to increased leaf size in BnATS1-OE plants.

A

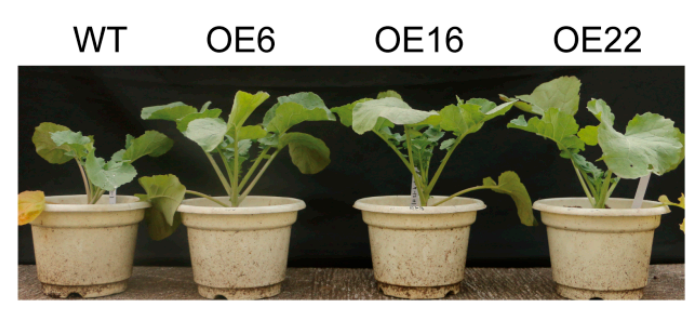

D

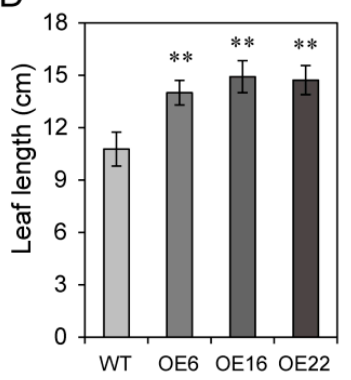

E

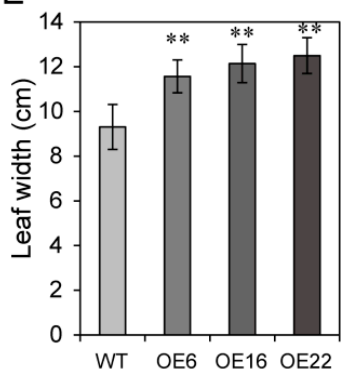

$\mathrm{B}$

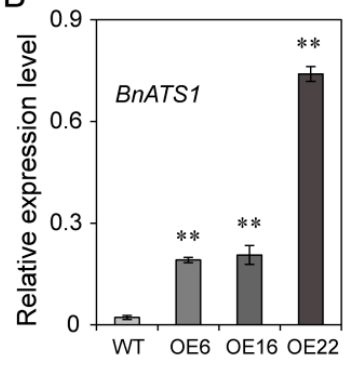

$\mathrm{F}$

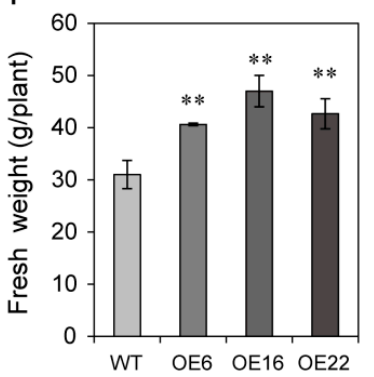

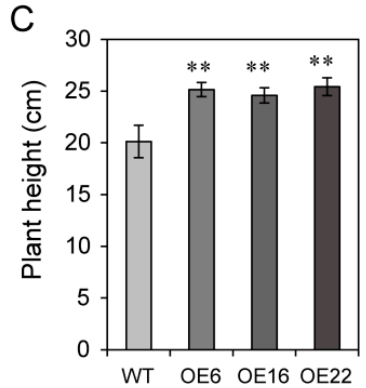

G

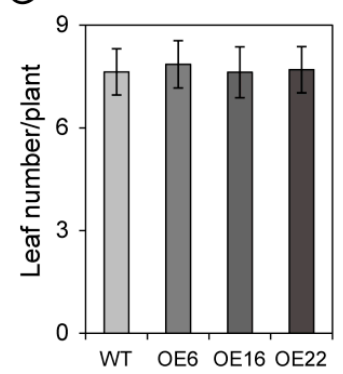

Figure 3. Overexpression of BnATS1 promoted plant growth at the vegetative stage. (A) The growth phenotype. (B) BnATS1 was overexpressed in cv. Westar plants as compared with the wild type (WT) plants detected by RT-qPCR. Total RNA was extracted from leaves of plants at the vegetative stage. $\beta$-Actin from Brassica napus was used as an internal standard. Values are mean $\pm \mathrm{SD}(n=3$ separate samples). (C) Plant height, (D) leaf length, (E) leaf width, (F) fresh weight of aboveground parts, and (G) leaf number of 26-day-old plants under field growth conditions. The leaf size was measured in the top 1st and 2nd leaves fully expanded in 26-day-old plants. The plant height represents the length of aboveground parts. Values are means $\pm \mathrm{SD}\left(n=5\right.$ independent experiments). ${ }^{* *}$ indicates significant difference at $p<0.01$ compared with the WT based on Student's $t$ test.

\subsection{The Effect of BnATS1 on Phospholipid Metabolism}

The results from the subcellular localization and activity assay suggest that BnATS1 catalyzes the first step of glycerolipid assembly to produce LPA in the prokaryotic pathway. To get insight into how BnATS1 affects plant growth, we profiled polar glycerolipid contents and lipid species from leaves of two representative BnATS1-OE lines, OE6 and OE16, compared with WT plants at the vegetative growth stage. Lipids from leaves were quantitatively analyzed by electron-spray ionization tandem mass spectrometry (ESI-MS/MS). PA content in leaves was very low at $0.129 \mathrm{nmol} / \mathrm{mg} \mathrm{DW}$, representing $0.19 \%$ of the total polar glycerolipids examined in WT leaves of $\mathrm{cv}$. Westar (Brasscia napus), and total PA content in BnATS1-OE leaves was not significantly different from that of WT plants (Figure 4). The molecular species including 34:3-, 34:2-, 36:6-, and 36:5-PA are major constituents of PA. The contents of most PA species in BnATS1-OE plants were not significantly different from that of the WT except higher 36:6- and 36:5-PA in OE6 and a lower 34:3-PA in OE16 relative to the WT (Figure 5). The results implicate that PA is rapidly transferred to DAG and CDP-DAG for other glycerolipid synthesis.

PG is the only major phospholipid in thylakoid membranes, which is mainly produced in the prokaryotic pathway in plants [4]. Lipid profiling showed that PG content was $6.8 \%$ of the total polar glycerolipids examined in WT leaves of cv. Westar. The major molecular species of PG were 34:4-, 34:3-, and 34:2-PG. Overexpression of BnATS1 led to an increased PG content compared with the WT (Figure 4). Increased PG in BnATS1-OE leaves resulted from increases in 34:3-, 34:2-, and 34:1-PG, 
which were partially coincided with those major species of PA (Figure 5). The results suggest that increased PG may be derived from PA mediated by BnATS1 overexpression.

$\mathrm{PC}$ is a major phospholipid in cellular membranes and is originally derived from PA via the CDP-choline pathway, and PC can be also produced via the PE methylation pathway [1]. PC content was approximately $15 \%$, whereas other phospholipids such as PE, PI, and phosphatidylserine (PS) were only $8.2 \%, 4.5 \%$, and $0.4 \%$, respectively, of the total polar glycerolipids examined in WT leaves of cv. Westar (Figure 4). PC in BnATS1-OE plants was increased by $34-43 \%$, compared with the WT (Figure 4). LysoPC (LPC) content in BnATS1-OE was also higher than that of the WT. Molecular profiling revealed that PC was predominantly composed of 34:3-, 34:2-, 36:6-, 36:5-, 36:4-, and 36:3-PC species. Elevated PC in BnATS1-OE leaves can be attributed to increases in almost all PC species, including 34:4-, 34:3-, 34:2-, 34:1-, 36:6-, 36:5-, 36:4-, 36:3-,36:2-, 36:1-, 38:6-, 38:5-, 38:4-, and 38:3-PC (Figure 5). In addition, PI content in BnATS1-OE plants was also higher than that of the WT, which was caused by elevations in 34:3- and 34:2-PI (Figures 4 and 5). By comparison, overexpression of BnATS1 did not lead to changes in PE and PS levels as compared to the WT (Figure 4). The results suggest that increased BnATS1 expression also promotes PC and PI production with increased polyunsaturated fatty acid species.

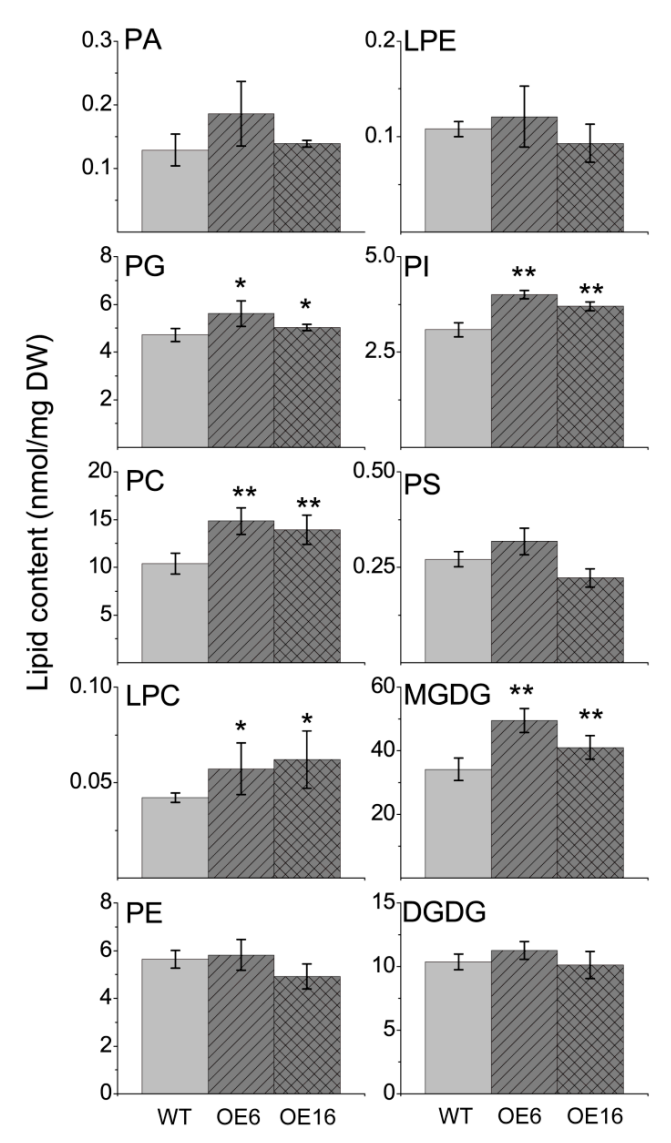

Figure 4. Effect of BnATS1-overexpression on membrane lipids. Lipids were extracted from leaves of 40-day-old plants under field growth conditions. Values are means $\pm S D$ $(n=4$ separate samples). * and ** denote significance at $p<0.05$ and $p<0.01$, respectively, compared with WT plants based on Student's $t$ test. PA, phosphatidic acid; PG, phosphatidylglycerol; PC, phosphatidylcholine; LPC, lysophosphatidylcholine; PE, phosphatidylethanolamine; LPE, lysophosphatidylethanolamine; PI, phosphatidylinositol; PS, phosphatidylserine; MGDG, monogalactosyldiacylglycerol; DGDG, digalactosyldiacylglycerol. 


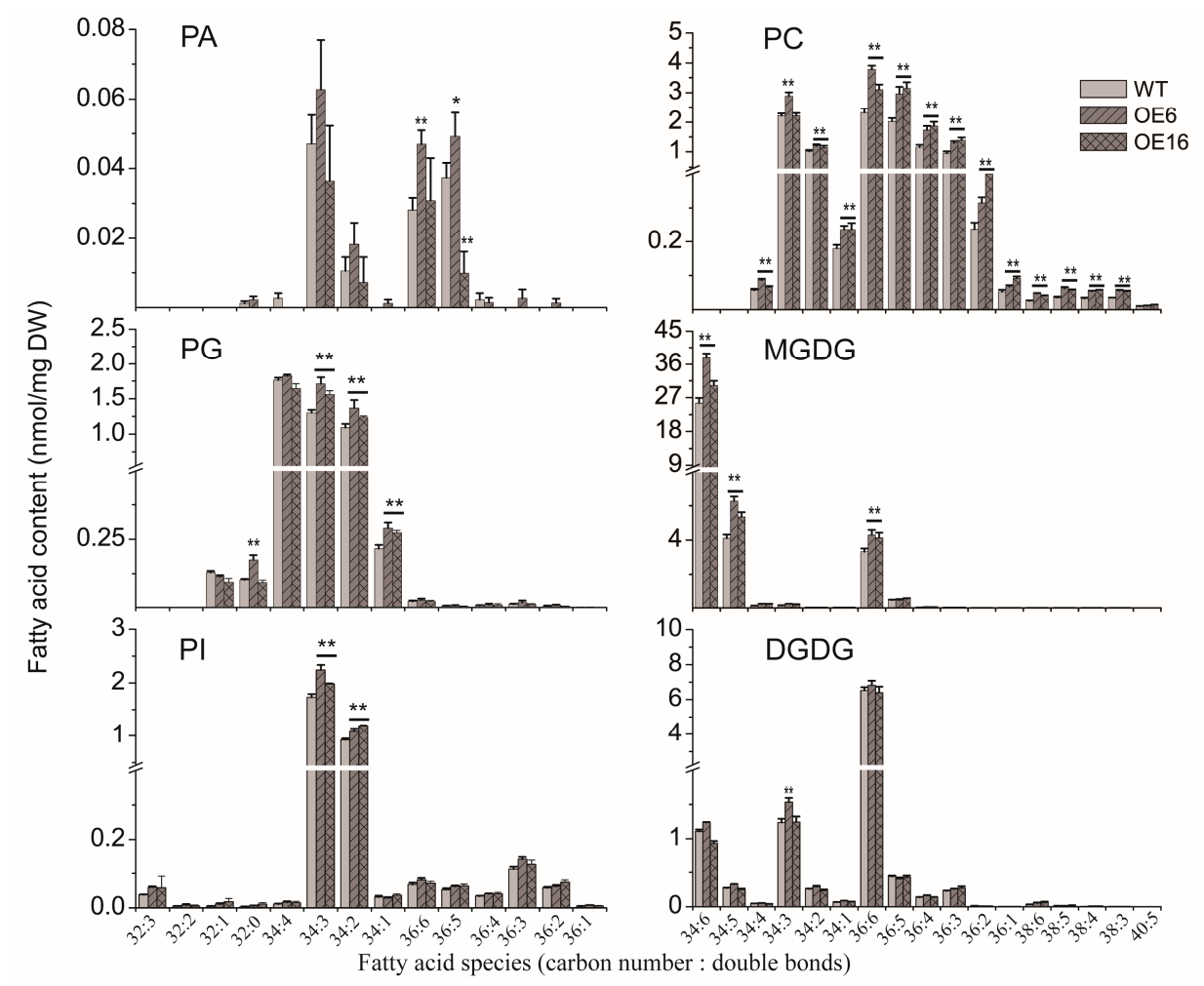

Figure 5. Effect of BnATS1-overexpression on membrane lipid composition. Lipids were extracted from leaves of 40-day-old plants under field growth conditions. Fatty acid species are shown as total acyl carbons: total double bonds. Values are means $\pm \operatorname{SD}\left(n=4\right.$ separate samples). ${ }^{*}$ and ${ }^{* *}$ denote significant difference at $p<0.05$ and $p<0.01$, respectively, compared with WT plants based on Student's $t$ test.

\subsection{Overexpression of BnATS1 Enhanced Prokaryotic Galactolipids}

Galactolipids, such as MGDG and DGDG, are mostly abundant in green tissues, particularly in leaves of plants. MGDG and DGDG were $49.6 \%$ and $15.1 \%$, respectively, of the total polar glycerolipids examined in WT leaves of cv. Westar (Figure 4). Lipid profiling showed that molecular species of MGDG were distinguishable from those in DGDG. MGDG in leaves were predominantly composed of 34:6-MGDG (18:3/16:3-MGDG), comprising 75\% of the total MGDG species, whereas DGDG was mainly composed of 36:6-DGDG (18:3/18:3-DGDG), comprising 63\% of the total DGDG species in WT leaves (Figure 5). The results suggest that MGDG is mainly assembled through the prokaryotic pathway in Brassica napus. Moreover, overexpression of BnATS1 led to a significant increase in MGDG. The MGDG contents in OE6 and OE16 leaves were increased by $45 \%$ and $20 \%$, respectively, as compared with WT plants. The increased MGDG in BnATS1-OE plants was caused predominantly by the elevations in 34:634:5-, and 36:6-MGDG (Figure 5). Although overexpression of BnATS1 did not affect the total DGDG content, 34:3-DGDG in the OE6 line was higher than that of the WT (Figure 5). These results suggest that enhanced thylakoid lipids in BnATS1-OE plants are due to enhanced galactolipid synthesis via the prokaryotic pathway mediated by BnATS1.

\subsection{Overexpression of BnATS1 Up-Regulated the Expression of Genes Related to Lipid Anabolism}

Lipid profiling showed that overexpression of BnATS1 promoted simultaneously the accumulation of multiple lipids, such as PG, MGDG, PC, and PI. It is possible that overexpression of BnATS1 may affect the expression of genes involved in different lipid metabolic processes. To test this possibility, RNA was extracted from the leaves of BnATS1-OE and WT plants and analyzed by real-time PCR. The $\beta$-ketoacyl-ACP reductase (KAR) is required for de novo FA synthesis [35], and the KAR expression level in BnATS1-OE leaves was two-fold higher than that of the WT (Figure 6). ATS2 catalyzes the 
sn-2 acylation of the glycerol backbone to produce PA in the prokaryotic pathway following ATS1 action [33], and the ATS2 transcript level in BnATS1-OE leaves was increased two-fold compared with the WT (Figure 6). Moreover, overexpression of BnATS1 led to up-regulation of genes, such as PEAMT (phosphoethanolamine N-methyltransferase) and AAPT1 (aminoalcoholphosphotransferase 1), involved in PC synthesis from DAG, and LPCAT (lysophosphatidylcholine acyltransferase) in PC-acyl editing. In addition, overexpression of BnATS1 also promoted the expression of PGPS (phosphatidylglycerolphosphate synthase) involved in PG synthesis. The PGPS expression was significantly up-regulated in OE6 and slightly increased in OE16 as compared with the WT (Figure 6). Taken together, the results suggest that overexpression of BnATS1 is able to promote synchronously the expression of genes involved in multiple lipid anabolic processes, thus coordinates with other enzymes to enhance glycerolipid synthesis in Brassica napus.
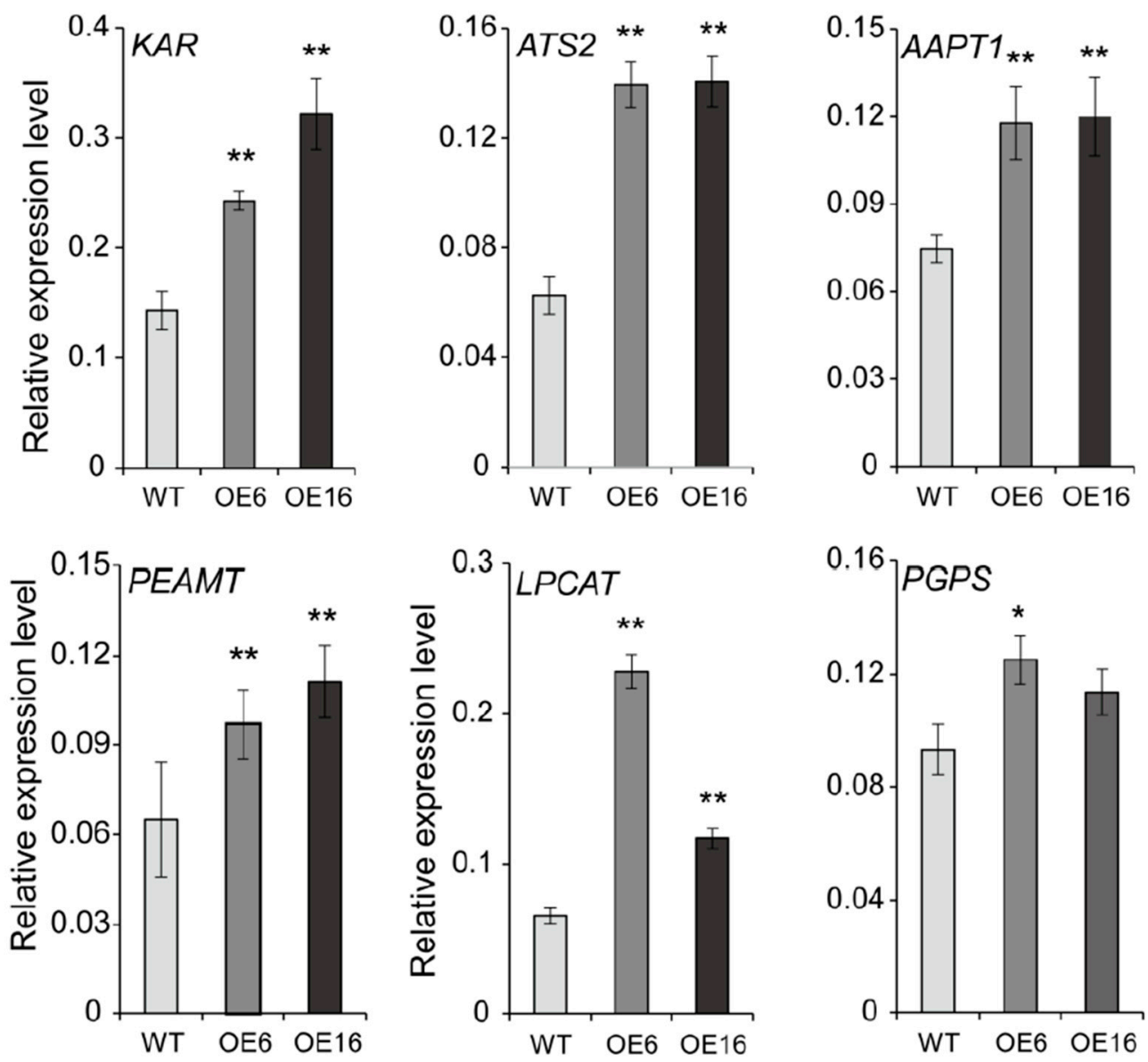

Figure 6. Overexpression of BnATS1 enhanced expression of genes related to the lipid anabolic process. Total RNA was extracted from leaves of 40-day-old plants under field growth conditions. The relative expression levels of genes were analyzed by real-time PCR normalized to the $\beta$-Actin expression level. Values are means $\pm \mathrm{SD}$ ( $n=3$ separate samples). * and ${ }^{* *}$ denote significance at $p<0.05$ and $p<0.01$, respectively, compared with WT plants based on Student's $t$ test.

\section{Discussion}

In plants, glycerolipids are assembled via two parallel pathways, the plastid-localized prokaryotic pathway and the ER-localized eukaryotic pathway. ATS1 catalyzes a committed step reaction in the prokaryotic pathway. The Arabidopsis mutant ats1 (also designated atc1) with defective ATS1 exhibits a reduction in prokaryotic thylakoid lipids [3,4]. However, the effect of overexpressing ATS1 on plant growth and lipid metabolism remained to be elucidated. Here, we found that plastid-localized 
BnATS1 in Brassica napus has a positive impact on plant growth, accompanied by increases in multiple glycerolipids including PG, MGDG, PC, and PI.

Our results from Brassica napus plants overexpressing BnATS1 showed that BnATS1 has a promotion on vegetative growth, particularly leaves. Indeed, BnATS1 was highly expressed in green tissues, being the highest in young leaves, suggesting its role in leaf growth. Our observation showed that BnATS1 is localized in chloroplasts, implicating its involvement in lipid assembly in the prokaryotic pathway. Fatty acids are synthesized de novo in chloroplasts [2], and this plastid-localized BnATS1 may facilitate the access of acyl groups for glycerolipid assembly. It was showed that ATS1 catalyzes the sn-1 acylation of G3P using 18:1-ACP and 16:0-ACP as substrates in Helianthus annuus [34]. Our result from the in vitro assay showed that BnATS1 is capable of using 16:0-CoA as a substrate to produce LPA, which is a precursor for PA synthesis through the acylation at the sn-2 of glycerol backbone. $\mathrm{PA}$ is an intermediate in glycerolipid metabolism and is rapidly transferred to other lipids $[1,2,36]$. PA produced in the prokaryotic pathway is responsible for synthesis of PG and galactolipids [15,16], whereas PA produced in the eukaryotic pathway is primarily involved in phospholipid synthesis, and could be transported to chloroplasts for galactolipid synthesis [1,17-19]. Our results from the lipid profiling showed that most PA species in BnATS1-OE plants were not significantly higher than in the WT. Instead, overexpression of BnATS1 led to increased PG in leaves. The increased molecular species of PG were mostly similar to those species abundant in PA, implicating that increased PG in BnATS1-OE plants may be derived from PA. PG is an essential component of photosynthetic membranes and most PG (approximately $85 \%$ of total PG) in plastids is derived from the prokaryotic pathway in Arabidopsis [37]. Increased PG in BnATS1-OE plants agrees with a previous report showing that the ats1 (act1) mutant had marked reduction in PG [3].

Galactolipids, such as MGDG and DGDG, are most abundant in land plants, which predominantly present in thylakoid membranes and are essential for photoautotrophic growth [12,38,39]. In Arabidopsis, MGDG in leaves is composed of comparable proportions between eukaryotic species (18:3/18:3-MGDG) and prokaryotic 18:3/16:3-MGDG, whereas DGDG is mostly composed of eukaryotic species (18:3/18:3-DGDG) [37]. Our lipid profiling revealed that MGDG in the leaves of Brassica napus was predominantly composed of 34:6-MGDG (18:3/16:3-MGDG) derived from the prokaryotic pathway, whereas DGDG is mainly 36:6-DGDG (18:3/18:3-DGDG) originated from the eukaryotic pathway. Moreover, BnATS1-OE plants exhibited an increased MGDG with elevated 34:6- and 34:5-MGDG, as compared with the WT, but the DGDG in BnATS1-OE plants is similar to that of the WT. The results suggest BnATS1 contributes to prokaryotic galactolipid accumulation, which agrees with previous reports showing that ats1 mutant deficiency is in the prokaryotic pathway [3,4]. In addition, BnATS1-OE plants had an increased 36:6-MGDG, which may be derived from increased 36:6-PC. PC, a major site of fatty acid desaturation, provides a DAG moiety with polyunsaturated acyl groups for eukaryotic galactolipids $[1,2]$.

Overexpression of BnATS1 also led to increased PC, which can be attributed to all molecular species of PC examined in leaves, as compared with the WT. PC is a major phospholipid in cellular membranes and an entry point for newly synthesized fatty acids from chloroplasts to the ER for glycerolipid assembly [40]. Our lipid profiling revealed that PC is mostly composed of polyunsaturated fatty acids such as 36:6-, 36:5-, 36:4, 36:3-, 34:3-, and 34:2-PC, and overexpression of BnATS1 increased PC with polyunsaturated acyl groups. The ratio of polyunsaturated to saturated fatty acids of PC in BnATS1-OE plants (4.7-5.5:1) was substantially higher than that of WT plants (3.9:1). It was reported that plant species with a higher content of unsaturated fatty acids exhibit more tolerance to cold stress than those species containing a higher level of saturated fatty acids [41]. Brassica napus plants frequently experience cold stress under the growth conditions in the field during winter. Increased PC with polyunsaturated acyl groups in BnATS1-OE plants may contribute to enhanced tolerance to cold stress, thus promoting plant growth under low-temperature conditions.

However, increased PC in BnATS1-OE plants may not be resulting directly from increased BnATS1 expression, as PA produced in the prokaryotic pathway may be not involved in PC synthesis [2,3]. 
In agreement with these alterations, overexpression of BnATS1 promotes the expression of genes such as KAR, ATS2, PEAMT, AAPT1, and LPCAT involved in de novo fatty acid synthesis, PC assembly, and acyl editing. In PC synthesis from the CDP-choline pathway, phosphoethanlamine is methylated to phosphocholine by PEAMT, and then is activated to CDP-choline [1,42]. The phosphocholine group of CDP-choline is further transferred to DAG by AAPT, producing PC [1,43]. LPCAT is involved in acyl editing through re-esterification of lyso-PC [10]. Our data showed that PC was largely increased in BnATS1-OE, which agrees with the increased expression of multiple genes in the lipid anabolic pathway. These results suggest that chloroplast-localized BnATS1 provides a "pull" force to enhance fatty acid synthesis and a "push" effect on glycerolipid assembly.

\section{Materials and Methods}

\subsection{Plant Materials and Growth Conditions}

The seeds of canola cultivar (cv.) Westar (Brassica napus L.) were germinated in pots containing soil. Two-week-old seedlings with comparable size were transferred to pots (one plant per pot) and grown with regular watering in field conditions from late autumn through the spring season in Wuhan, China. The temperature was approximately $7-10{ }^{\circ} \mathrm{C}$ (night) $/ 15-20{ }^{\circ} \mathrm{C}$ (day) in late autumn and $0-3{ }^{\circ} \mathrm{C}$ (night) $/ 7-12{ }^{\circ} \mathrm{C}$ (day) in winter in Wuhan, China.

\subsection{Gene Cloning, Vector Construction and Plant Transformation}

A cDNA pool was synthesized from mRNA extracted from leaves of cv. Westar (Brassica napus) plants by reverse transcription using a TransScript cDNA Synthesis SuperMix Kit according to the manufacturer's instructions (TansGene Biotech, Beijing, China). The full-length BnATS1 cDNA was amplified from cDNA pools by PCR using the primers BnATS1-OE-F 5'-TCTAGAATGACTCTCACGTTTTCCTC-3' (forward) and BnATS1-OE-R $5^{\prime}$-GAGCTCCTAATTCCAAGGTTGTGACA-3' (reverse), and then ligated into the expression vector pBI121 after digestion with $\mathrm{XbaI}$ and $\mathrm{SacI}$ under the control of the $35 \mathrm{~S}$ promoter. The construct containing BnATS1 was confirmed by sequencing and then introduced into Agrobacterium tumefaciens strain GV3101, which was used to infect cv. Westar hypocotyls in tissue culture to get regeneration plants based on the methods described previously [44]. The transgenic plants were verified by PCR using the pBI121 vector sequence specific primer 5'-GATGGTTAGAGAGGCTTACGCA-3' and BnATS1 specific primer BnATS1-OE-R (Supplementary Table S1).

\subsection{RNA Extraction and Real-Time PCR}

Total RNA was isolated from various tissues of cv. Westar plants using Transzol reagent (TransGen Biotech, Beijing, China). RNA extracts were treated with DNaseI to remove contaminating DNA and used as a template to synthesize the first-strand cDNA by reverse transcription using a TransScript cDNA Synthesis SuperMix Kit according to the manufacturer's instructions (TransGen Biotech, Beijing, China). $\beta$-Actin from Brassica napus was used as an internal standard. Real-time PCR was performed as described previously [45]. The primers used are listed in Supplementary Table S1.

\subsection{BnATS1 Protein Expression and Activity Assay}

The full-length BnATS1 coding sequence (CDS) omitting the stop codon was amplified by PCR using primers BnATS1-PF and BnATS1-PR (Supplementary Table S1) and then was cloned into the pET28a vector at the cutting sites of EcoRI and XhoI. After sequence confirmation, the construct was introduced into the E. coli Rosetta (DE3) strain (TransGen Biotech, Beijing, China) and cultured in LB liquid medium until the $\mathrm{OD}_{600}$ reached 0.6 for protein expression by the induction with $0.5 \mathrm{mM}$ isopropyl $\beta$-D-thiogalactopyranoside (IPTG) for $20 \mathrm{~h}$ at $16^{\circ} \mathrm{C}$. Cells were lysed by ultrasonification in cold lysis buffer (50 mM Tris-HCl, pH 7.5, $120 \mathrm{mM} \mathrm{NaCl}, 1 \mathrm{mM} \mathrm{DTT}, 0.1 \%$ Triton-100, 10\% glycerol), and followed by centrifugation at $10,000 \mathrm{~g}$ at $4{ }^{\circ} \mathrm{C}$ for $20 \mathrm{~min}$. BnATS1 activity was assayed in $100 \mu \mathrm{L}$ 
of reaction mixtures containing $250 \mu \mathrm{M}$ HEPES buffer (pH 7.4), $8 \mu \mathrm{M}$ 16:0-CoA, $600 \mu \mathrm{M}$ G3P-Ca, $5 \mathrm{mg} / \mathrm{mL} \mathrm{BSA}$, and $20 \mu \mathrm{g}$ crude proteins incubated at $25^{\circ} \mathrm{C}$ for $5 \mathrm{~min}$. The reaction was stopped by addition of $300 \mu \mathrm{L}$ of chloroform: methanol $(1: 2, v / v)$. Resultant lipids were separated on thin layer chromatography (TLC) plates using a developing solvent (chloroform: methanol: acetic acid: $\left.\mathrm{H}_{2} \mathrm{O}=85: 15: 10: 3.5, v / v\right)$ and were visualized with iodine vapor [34]. The spots corresponding to LPA were quantified by the Image J software (1.48, National Institutes of Health, USA).

\subsection{Subcellular Localization}

The full-length BnATS1 CDS was amplified by PCR using primers BnATS1-GFP-F 5'-GGTACCA TGACTCTCACGTTTTCCTC-3' (forward) and BnATS1-GFP-R $5^{\prime}$-TCTAGAATTCCAAGGTTGTGACA AAG-3' (reverse) and cloned into the pCAMBIA1301s vector after digestion with KpnI and XbaI, which results in in-frame C-terminal fusion to GFP. The construct was introduced into Agrobacterium tumefaciens GV3101. The transformant was grown overnight in liquid LB media, and then centrifuged at $4000 \mathrm{rpm}$ for $10 \mathrm{~min}$. The cells were resuspended with the solution containing $10 \mathrm{mM} \mathrm{MgCl}_{2}$ and $1 \mathrm{mM}$ acetosyringone, and used for infiltrating leaves of 4-week-old tobacco (Nicotiana benthamiana) plants for transient expression driven by the 35S promoter. After infection for 2 to 3 days, BnATS1-GFP was visualized using a confocal laser scanning microscope (Leica, Biberach, Germany).

\subsection{Lipid Extraction and Analysis}

Total lipids were extracted according to the method described previously [46]. Briefly, leaf discs ( $0.5 \mathrm{~g}$ per sample) were sampled from 40-day-old plants (cv. Westar) and immediately immersed in $75^{\circ} \mathrm{C}$ isopropanol $(5 \mathrm{~mL})$ containing $0.01 \%$ butylated hydroxytoluene (BHT) for $15 \mathrm{~min}$ to arrest enzymatic activity. After cooling to room temperature $\left(25-28{ }^{\circ} \mathrm{C}\right)$, the sovlent $(3.5 \mathrm{~mL})$ of chloroform/water $(2.5: 1$, $v / v)$ was added to the sample and incubated for $1 \mathrm{~h}$ at room temperature by shaking. The extracts were transferred to clean glass tubes, and then the remaining leaves were re-extracted with $5 \mathrm{~mL}$ chloroform/methanol $(2: 1, v / v)$ containing $0.01 \%$ BHT at room temperature for several times until leaf tissues became bleached. The extracts were combined and washed twice with $1 \mathrm{M} \mathrm{KCl}$ and once with water, and dried under a stream of nitrogen gas, and then dissolved in a defined volume of chloroform. Lipids were quantitatively profiled by electron-spray ionization tandem mass spectrometry (ESI-MS/MS) based on the approach described previously [46].

Supplementary Materials: The following are available online at http://www.mdpi.com/1422-0067/21/15/5325/s1, Figure S1: Glycerolipid synthesis in two parallel pathways, Table S1: Primers Used in This Study.

Author Contributions: Investigation, H.K., C.J., N.L., A.A.A.A., W.C., W.G. and S.T.; Supervision, Y.H. All authors have read and agreed to the published version of the manuscript.

Funding: This work was funded by the National Key Basic Research Program of China (2015CB150204).

Conflicts of Interest: The authors declare no conflict of interest.

\section{References}

1. Li-Beisson, Y.H.; Shorrosh, B.; Beisson, F.; Andersson, M.X.; Arondel, V.; Bates, P.D.; Baud, S.; Bird, D.; DeBono, A.; Durrett, T.P.; et al. Arabidopsis Book; Robt, L., Ed.; The American Society of Plant Biologists: Rockville, MD, USA, 2013; Volume 11, pp. 1-70.

2. Ohlrogge, J.; Browse, J. Lipid biosynthesis. Plant Cell 1995, 7, 957-970. [PubMed]

3. Kunst, L.; Browse, J.; Somerville, C. Altered regulation of lipid biosynthesis in a mutant of Arabidopsis deficient in chloroplast glycerol-3-phosphate acyltransferase activity. Proc. Natl. Acad. Sci. USA 1988, 85, 4143-4147. [CrossRef] [PubMed]

4. Xu, C.; Yu, B.; Cornish, A.J.; Froehlich, J.E.; Benning, C. Phosphatidylglycerol biosynthesis in chloroplasts of Arabidopsis mutants deficient in acyl-ACP glycerol-3-phosphate acyltransferase. Plant J. 2006, 47, 296-309. [CrossRef] [PubMed] 
5. Xue, H.W.; Hosaka, K.; Plesch, G.; Mueller-Roeber, B. Cloning of Arabidopsis thaliana phosphatidylinositol synthase and functional expression in the yeast pis mutant. Plant Mol. Biol. 2000, 42, 757-764. [CrossRef] [PubMed]

6. Babiychuk, E.; Muller, F.; Eubel, H.; Braun, H.P.; Frentzen, M.; Kushnir, S. Arabidopsis phosphatidylglycerophosphate synthase 1 is essential for chloroplast differentiation, but is dispensable for mitochondrial function. Plant J. 2003, 33, 899-909. [CrossRef] [PubMed]

7. Lofke, C.; Ischebeck, T.; Konig, S.; Freitag, S.; Heiliviann, I. Alternative metabolic fates of phosphatidylinositol produced by phosphatidylinositol synthase isoforms in Arabidopsis thaliana. Biochem. J. 2008, 413, 115-124. [CrossRef]

8. Nakamura, Y.; Awai, K.; Masuda, T.; Yoshioka, Y.; Takamiya, K.-i.; Ohta, H. A novel phosphatidylcholine-hydrolyzing phospholipase $\mathrm{C}$ induced by phosphate starvation in Arabidopsis. J. Biol. Chem. 2005, 280, 7469-7476. [CrossRef]

9. Craddock, C.P.; Adams, N.; Bryant, F.M.; Kurup, S.; Eastmond, P.J. PHOSPHATIDIC ACID PHOSPHOHYDROLASE regulates phosphatidylcholine biosynthesis in Arabidopsis by phosphatidic acid-mediated activation of CTP: PHOSPHOCHOLINE CYTIDYLYLTRANSFERASE activity. Plant Cell 2015, 27, 1251-1264. [CrossRef]

10. Bates, P.D.; Fatihi, A.; Snapp, A.R.; Carlsson, A.S.; Browse, J.; Lu, C.F. Acyl editing and headgroup exchange are the major mechanisms that direct polyunsaturated fatty acid flux into triacylglycerols. Plant Physiol. 2012, 160, 1530-1539. [CrossRef]

11. Bates, P.D.; Ohlrogge, J.B.; Pollard, M. Incorporation of newly synthesized fatty acids into cytosolic glycerolipids in pea leaves occurs via acyl editing. J. Biol. Chem. 2007, 282, 31206-31216. [CrossRef]

12. Kobayashi, K.; Kondo, M.; Fukuda, H.; Nishimura, M.; Ohta, H. Galactolipid synthesis in chloroplast inner envelope is essential for proper thylakoid biogenesis, photosynthesis, and embryogenesis. Proc. Natl. Acad. Sci. USA 2007, 104, 17216-17221. [CrossRef] [PubMed]

13. Cases, S.; Smith, S.J.; Zheng, Y.W.; Myers, H.M.; Lear, S.R.; Sande, E.; Novak, S.; Collins, C.; Welch, C.B.; Lusis, A.J.; et al. Identification of a gene encoding an acyl coa:diacylglycerol acyltransferase, a key enzyme in triacylglycerol synthesis. Proc. Natl. Acad. Sci. USA 1998, 95, 13018-13023. [CrossRef] [PubMed]

14. Nakamura, Y.; Koizumi, R.; Shui, G.; Shimojima, M.; Wenk, M.R.; Ito, T.; Ohta, H. Arabidopsis lipins mediate eukaryotic pathway of lipid metabolism and cope critically with phosphate starvation. Proc. Natl. Acad. Sci. USA 2009, 106, 20978-20983. [CrossRef] [PubMed]

15. Xu, C.; Fan, J.; Riekhof, W.; Froehlich, J.E.; Benning, C. A permease-like protein involved in ER to thylakoid lipid transfer in Arabidopsis. EMBO J. 2003, 22, 2370-2379. [CrossRef] [PubMed]

16. Xu, C.; Fan, J.; Froehlich, J.E.; Awai, K.; Benning, C. Mutation of the tgd1 chloroplast envelope protein affects phosphatidate metabolism in Arabidopsis. Plant Cell 2005, 17, 3094-3110. [CrossRef] [PubMed]

17. Cruz-Ramírez, A.; Oropeza-Aburto, A.; Razo-Hernandez, F.; Ramirez-Chavez, E.; Herrera-Estrella, L. Phospholipase DZ2 plays an important role in extraplastidic galactolipid biosynthesis and phosphate recycling in Arabidopsis roots. Proc. Natl. Acad. Sci. USA 2006, 103, 6765-6770. [CrossRef]

18. Li, M.; Welti, R.; Wang, X. Quantitative profiling of Arabidopsis polar glycerolipids in response to phosphorus starvation. Roles of phospholipases $\mathrm{D} \zeta 1$ and $\mathrm{D} \zeta 2$ in phosphatidylcholine hydrolysis and digalactosyldiacylglycerol accumulation in phosphorus-starved plants. Plant Physiol. 2006, 142, 750-761. [CrossRef]

19. Gaude, N.; Nakamura, Y.; Scheible, W.; Ohta, H.; Dormann, P. Phospholipase c5 (npc5) is involved in galactolipid accumulation during phosphate limitation in leaves of Arabidopsis. Plant J. 2008, 56, 28-39. [CrossRef]

20. Heinz, E.; Roughan, P.G. Similarities and differences in lipid metabolism of chloroplasts isolated from 18:3 and 16:3 plants. Plant Physiol. 1983, 72, 273-279. [CrossRef]

21. Zhu, S.Q.; Zhao, H.; Zhou, R.; Ji, B.H.; Dan, X.Y. Substrate selectivity of glycerol-3-phosphate acyltransferase in rice. J. Integr. Plant Biol. 2009, 51, 1040-1049. [CrossRef]

22. Zheng, Z.F.; Xia, Q.; Dauk, M.; Shen, W.Y.; Selvaraj, G.; Zou, J.T. Arabidopsis AtGPAT1, a member of the membrane-bound glycerol-3-phosphate acyltransferase gene family, is essential for tapetum differentiation and male fertility. Plant Cell 2003, 15, 1872-1887. [CrossRef] [PubMed]

23. Beisson, F.; Li, Y.H.; Bonaventure, G.; Pollard, M.; Ohlrogge, J.B. The acyltransferase GPAT5 is required for the synthesis of suberin in seed coat and root of Arabidopsis. Plant Cell 2007, 19, 351-368. [CrossRef] 
24. Li, Y.; Beisson, F.; Koo, A.J.K.; Molina, I.; Pollard, M.; Ohlrogge, J. Identification of acyltransferases required for cutin biosynthesis and production of cutin with suberin-like monomers. Proc. Natl. Acad. Sci. USA 2007, 104, 18339-18344. [CrossRef] [PubMed]

25. Li-Beisson, Y.H.; Pollard, M.; Sauveplane, V.; Pinot, F.; Ohlrogge, J.; Beisson, F. Nanoridges that characterize the surface morphology of flowers require the synthesis of cutin polyester. Proc. Natl. Acad. Sci. USA 2009, 106, 22008-22013. [CrossRef] [PubMed]

26. Yang, W.; Pollard, M.; Li-Beisson, Y.H.; Beisson, F.; Feig, M.; Ohlrogge, J. A distinct type of glycerol-3-phosphate acyltransferase with $s n-2$ preference and phosphatase activity producing 2-monoacylglycerol. Proc. Natl. Acad. Sci. USA 2009, 107, 12040-12045. [CrossRef] [PubMed]

27. Yang, W.; Simpson, J.P.; Li-Beisson, Y.H.; Beisson, F.; Pollard, M.; Ohlrogge, J.B. A land-plant-specific glycerol-3-phosphate acyltransferase family in Arabidopsis: Substrate specificity, sn-2 preference, and evolution. Plant Physiol. 2012, 160, 638-652. [CrossRef] [PubMed]

28. Men, X.; Shi, J.X.; Liang, W.Q.; Zhang, Q.F.; Lian, G.B.; Quan, S.; Zhu, L.; Lou, Z.J.; Chen, M.J.; Zhang, D.B. Glycerol-3-Phosphate Acyltransferase 3 (OsGPAT3) is required for anther development and male fertility in rice. J. Exp. Bot. 2017, 68, 513-526. [CrossRef]

29. Li, X.; Zhu, J.; Yang, J.; Zhang, G.R.; Xing, W.F.; Zhang, S.; Yang, Z.N. Glycerol-3-phosphate acyltransferase 6 (GPAT6) is important for tapetum development in Arabidopsis and plays multiple roles in plant fertility. Mol. Plant 2012, 5, 131-142. [CrossRef]

30. Petit, J.; Bres, C.; Mauxion, J.; Tai, F.; Martin, L.; Fich, E.A.; Joubes, J.; Rose, J.; Domergue, F.; Rothan, C. The glycerol-3-phosphate acyltransferase GPAT6 from tomato plays a central role in fruit cutin biosynthesis. Plant Physiol. 2016, 171, 894-913. [CrossRef]

31. Shockey, J.; Regmi, A.; Cotton, K.; Adhikari, N.D.; Browse, J.; Bates, P.D. Identification of Arabidopsis GPAT9 (At5g60620) as an essential gene involved in triacylglycerol biosynthesis. Plant Physiol. 2016, 170, 163-179. [CrossRef]

32. Singer, S.D.; Chen, G.Q.; Mietkiewska, E.; Tomasi, P.; Jayawardhane, K.; Dyer, J.M.; Weselake, R.J. Arabidopsis GPAT9 contributes to synthesis of intracellular glycerolipids but not surface lipids. J. Exp. Bot. 2016, 67, 4627-4638. [CrossRef] [PubMed]

33. Kim, H.U.; Huang, A.H.C. Plastid lysophosphatidyl acyltransferase is essential for embryo development in Arabidopsis. Plant Physiol. 2004, 134, 1206-1216. [CrossRef] [PubMed]

34. Payá-Milans, M.; Venegas-Calerón, M.; Salas, J.J.; Garces, R.; Martinez-Force, E. Cloning, heterologous expression and biochemical characterization of plastidial sn-glycerol-3-phosphate acyltransferase from Helianthus annuus. Phytochemistry 2015, 111, 27-36. [CrossRef]

35. Mou, Z.L.; He, Y.K.; Dai, Y.; Liu, X.F.; Li, J.Y. Deficiency in fatty acid synthase leads to premature cell death and dramatic alterations in plant morphology. Plant Cell 2000, 12, 405-417. [CrossRef]

36. Wang, X.; Devaiah, S.P.; Zhang, W.; Welti, R. Signaling functions of phosphatidic acid. Prog. Lipid Res. 2006, 45, 250-278. [CrossRef] [PubMed]

37. Browse, J.; Warwick, N.; Somerville, C.R.; Slack, C.R. Fluxes through the prokaryotic and eukaryotic pathways of lipid synthesis in the '16:3' plant Arabidopsis thaliana. Biochem. J. 1986, 235, 25-31. [CrossRef] [PubMed]

38. Dörmann, P.; Hoffmann-Benning, S.; Balbo, I.; Benning, C. Isolation and characterization of an Arabidopsis mutant deficient in the thylakoid lipid digalactosyl diacylglycerol. Plant Cell 1995, 7, 1801-1810.

39. Umena, Y.; Kawakami, K.; Shen, J.R.; Kamiya, N. Crystal structure of oxygen-evolving photosystem II at a resolution of $1.9 \AA$. Nature 2011, 473, 55-60. [CrossRef]

40. Tjellstrom, H.; Yang, Z.; Allen, D.K.; Ohlrogge, J.B. Rapid kinetic labeling of Arabidopsis cell suspension cultures: Implications for models of lipid export from plastids. Plant Physiol. 2012, 158, 601-611. [CrossRef]

41. Chen, M.; Thelen, J.J. ACYL-LIPID DESATURASE2 is required for chilling and freezing tolerance in Arabidopsis. Plant Cell 2013, 25, 1430-1444. [CrossRef]

42. Mou, Z.L.; Wang, X.Q.; Fu, Z.M.; Dai, Y.; Han, C.; Jian, O.Y.; Bao, F.; Hu, Y.X.; Li, J.Y. Silencing of phosphoethanolamine $\mathrm{N}$-methyltransferase results in temperature-sensitive male sterility and salt hypersensitivity in Arabidopsis. Plant Cell 2002, 14, 2031-2043. [CrossRef] [PubMed]

43. Qi, Q.; Huang, Y.F.; Cutler, A.J.; Abrams, S.R.; Taylor, D.C. Molecular and biochemical characterization of an aminoalcoholphosphotransferase (AAPT1) from Brassica napus: Effects of low temperature and abscisic acid treatments on AAPT expression in Arabidopsis plants and effects of over-expression of BnAAPT1 in transgenic Arabidopsis. Planta 2003, 217, 547-558. [PubMed] 
44. Lu, S.P.; Bahn, S.C.; Qu, G.; Qin, H.Y.; Hong, Y.; Xu, Q.P.; Zhou, Y.M.; Hong, Y.Y.; Wang, X.M. Increased expression of phospholipase $\mathrm{D} \alpha 1$ in guard cells decreases water loss with improved seed production under drought in Brassica napus. Plant Biotechnol. J. 2013, 11, 380-389. [CrossRef] [PubMed]

45. Hong, Y.; Yuan, S.; Sun, L.X.; Wang, X.M.; Hong, Y.Y. Cytidinediphosphate-diacylglycerol synthase 5 is required for phospholipid homeostasis and is negatively involved in hyperosmotic stress tolerance. Plant $J$. 2018, 94, 1038-1050. [CrossRef] [PubMed]

46. Welti, R.; Li, W.Q.; Li, M.Y.; Sang, Y.M.; Biesiada, H.; Zhou, H.; Rajashekar, C.B.; Williams, T.D.; Wang, X.M. Profiling membrane lipids in plant stress responses. Role of phospholipase D alpha in freezing-induced lipid changes in Arabidopsis. J. Biol. Chem. 2002, 277, 31994-32002. [CrossRef]

(C) 2020 by the authors. Licensee MDPI, Basel, Switzerland. This article is an open access article distributed under the terms and conditions of the Creative Commons Attribution (CC BY) license (http://creativecommons.org/licenses/by/4.0/). 